\title{
Effect of Channel Envelope Synchrony on Interaural Time Difference Sensitivity in Bilateral Cochlear Implant Listeners
}

\author{
Tom Francart, ${ }^{1}$ Anneke Lenssen, ${ }^{1}$ Andreas Büchner, ${ }^{2}$ Thomas Lenarz, ${ }^{2}$ and Jan Wouters ${ }^{1}$
}

\begin{abstract}
Objectives: For a periodic acoustic input signal, the channel envelopes coded by current bilateral cochlear implant sound processors can be asynchronous. The effect of this asynchrony on sensitivity to interaural time differences (ITDs) was assessed.
\end{abstract}

Design: ITD sensitivity was measured in six bilateral cochlear implant listeners for single- and three-electrode stimuli. The three-electrode stimuli contained envelope modulations, either synchronous or asynchronous across electrodes, with delays of 1.25 up to $5.00 \mathrm{~ms}$. Each individual electrode carried the same ITD. Either neighboring electrodes were chosen or a separation of four electrodes to investigate the effect of electrode distance.

Results: With synchronous envelopes, no difference in ITD sensitivity was found among single-electrode, adjacent three-electrode, and spaced three-electrode stimuli. A decrease in ITD sensitivity was found with increasing across-channel envelope asynchrony, which was consistent with the use of the across-electrode aggregate stimulation pattern rather than individual information channels for ITDs. No consistent effect of electrode separation was found.

Conclusions: While the binaural system was resilient to small delays between envelopes, larger delays significantly deceased ITD sensitivity, both for adjacent and further spaced electrodes.

Key words: Bilateral, Binaural hearing, Channel envelope asynchrony, Cochlear implant, Interaural time difference.

(Ear \& Hearing 2015;36;e199-e206)

\section{INTRODUCTION}

Bilateral cochlear implants (CIs) have been shown to improve sound source localization and to improve speech intelligibility in noise in certain conditions (Ching et al. 2007; Schafer et al. 2011) compared with unilateral CIs. However, performance on these tasks remains much poorer for bilateral CI users than for normal-hearing $(\mathrm{NH})$ listeners. One of the main reasons for this discrepancy is the limited availability of interaural time differences (ITDs; Seeber \& Fastl 2008), which are important cues for sound source localization and binaural unmasking. The limited ITD availability is due to on the one hand the way current CI sound processors work and on the other hand perceptual limitations.

Current pairs of clinical sound processors do not clearly code ITDs, because (1) fine timing information is discarded, (2) processors are not synchronized across ears, and (3) signal modulations are not always sufficiently deep to allow ITD

${ }^{1}$ ExpORL, Department of Neurosciences, KU Leuven, Leuven, Belgium; and ${ }^{2}$ Department of Otolaryngology, Medical University of Hannover, Hannover, Germany. sensitivity (van Hoesel \& Tyler 2003; Ihlefeld et al. 2014). When these technical issues are circumvented by using optimal stimuli under laboratory conditions, ITD sensitivity, even for the best performers, is much poorer than for $\mathrm{NH}$ listeners using temporal fine structure ITD cues. In most studies with controlled electrical stimulation, single-electrode stimuli have been used (e.g., van Hoesel \& Tyler 2003; van Hoesel et al. 2009; Laback et al. 2011). ITD sensitivity varies widely across subjects, with the best discrimination thresholds around 50 to $100 \mu$ s and in the worst case no measurable ITD sensitivity at all.

Most studies so far have used stimuli consisting of either low-rate pulse trains or modulated high-rate pulse trains, and listeners can be sensitive to ITDs in both (Majdak et al. 2006; van Hoesel 2007; van Hoesel et al. 2009). In the remainder of this article, we will focus on modulated high-rate pulse trains because this is the type of stimulus used in most current sound processing strategies. The main stimulus-related factors influencing ITD thresholds are (1) modulation rate, with peak sensitivity at around $100 \mathrm{~Hz}$ (Noel \& Eddington 2013); (2) modulation depth, with improving sensitivity with increasing modulation depth (van Hoesel \& Tyler 2003; Ihlefeld et al. 2014); and (3) dead time (off-time) between modulation cycles, with improving sensitivity with increasing dead time (Laback et al. 2011). In summary, for single-electrode stimulation, it seems that best sensitivity can be achieved with deeply modulated pulse trains, a modulation frequency around $100 \mathrm{~Hz}$ and sufficient dead time between bursts of stimulation.

Clinical strategies developed for speech encoding use more complicated stimulation patterns than the simple single-electrode stimuli used in most ITD discrimination experiments. Ecological sounds, in particular speech, are broadband and require stimulation of more than one electrode. The interaction between ITDs encoded in the envelopes presented on more than one left-right electrode pair has not been thoroughly studied so far. Important remaining questions are (1) whether stimulation at multiple tonotopic places provides separate information channels for ITDs, and if so (2) how ITD information presented in multiple channels is combined by the auditory system.

With electric stimulation, especially in monopolar mode (current flows between an intracochlear electrode and extracochlear reference electrode), spread of excitation is broad, so electrodes that are close together stimulate the same population of neurons, leading to a reduced number of information channels available. This phenomenon clearly surfaces in various studies of interchannel delay discrimination (e.g., Tong \& Clark 1986) and pitch perception with electric stimulation (McKay \& McDermott 1996, 1999). However, a more recent study by Macherey and Carlyon (2010) showed that the pitch elicited by dual-channel 
pulse train stimuli on neighboring electrodes never matched the pitch elicited by the aggregate pattern on a single electrode. This suggests that there may be more independence between channels than previously thought, perhaps due to improved electrode technology. This prompted us to investigate this for bilateral CI ITD sensitivity.

A second factor that influences differences between singleversus multiple-electrode stimuli ITD sensitivity is, if multiple information channels are available, how binaural information in these channels is combined. The effect of interactions between the temporal envelopes in different frequency regions on the detection of ITD has been investigated in NH listeners (Buell et al. 1991; Stellmack 1992; Heller \& Trahiotis 1995). These studies report interference on the detection of a target ITD caused by a diotic stimulus on a remote frequency. Other studies, rather than investigating interference, assessed the effect of across frequency interactions on the integration of binaural information present in different frequency regions (Dye 1990; Buell \& Trahiotis 1993). They showed summation of binaural information across frequency: ITD sensitivity increased with increasing number of spectral components carrying the same ITD. Saberi (1995) investigated the effect of comodulation on ITD thresholds and found that comodulated bands produced lower ITD thresholds compared with independent bands. He also investigated the effect of starting phase for spectrally remote sinusoidally amplitude-modulated tones and found that ITD thresholds were lowest if the starting phase was the same. We will refer to this ensemble of effects as ITD summation, indicating improving ITD sensitivity when the same ITDs are present in tonotopically separate channels.

For multielectrode stimulation with bilateral CIs, the question is how the combination of spread of excitation, binaural interference, and ITD summation affects ITD sensitivity. Several studies have investigated ITD sensitivity with clinical processors, stimulating multiple electrodes (Laback et al. 2004; Senn et al. 2005; Grantham et al. 2008). Performance was generally lower with speech processors than with direct single-electrode stimulation. The influence of the speech processor is evident from the fact that ITD sensitivity was very stimulus dependent. Sensitivity with clinical processors was best for (acoustic) click trains, worse for noise bursts (Senn et al. 2005), and even worse for a speech fragment or modulated noise (Laback et al. 2004). Unfortunately, these studies do not allow to identify which aspects of the stimulation patterns cause the sensitivity decrease compared with controlled single-electrode stimulation.

Most clinical CI sound processors function by sending the broadband acoustic signal through a filter bank and applying envelope detection, discarding temporal fine structure. The resulting envelopes are used to modulate interleaved fixed-rate pulse trains. For a modulated acoustic input signal, such as a spoken vowel, the resulting envelopes are often not modulated in phase due to four main factors. (1) Only one electrode is stimulated at a time, so adjacent channel envelopes will always be delayed with each other, by at least the period of the total pulse rate, for example, $139 \mu$ s for a total pulse rate of 7200 pulses per second ( 8 maxima times 900 pulses per second, which is the default stimulation rate in the system produced by Cochlear). (2) Due to differences in group delay between filters in the filter bank used. The magnitude of this effect depends strongly on the filter bank implementation. (3) Due to the effect of room acoustics on the input signal. (4) Due to maxima selection: when a number of channel envelopes are modulated simultaneously, and other channels are not, at the maximum amplitude of the modulation the modulated channels will be selected, while at the minimum the others will be selected. This leads to modulation of the channels which were not originally modulated with a delay of half the period of the modulation, for example, for vowel with a fundamental frequency of $100 \mathrm{~Hz}$, a delay of $5 \mathrm{~ms}$ can be obtained.

In the present study, we investigated the effect on ITD sensitivity of three stimulus parameters: (1) single versus three-electrode, (2) channel envelope (a)synchrony, and (3) electrode separation. In the three-electrode stimulus, the same ITD was represented by the three-channel envelopes on corresponding left-right electrode pairs. To investigate the effect of envelope (a)synchrony, we delayed the stimulus on one or two left-right electrodes pairs by varying amounts, ranging from 1.25 to $5 \mathrm{~ms}$, corresponding to the magnitude of the effects described earlier for clinical processors. We then measured the effect of delay on ITD sensitivity. If the three electrodes represent independent information channels, ITD summation would be expected, leading to improved ITD sensitivity, as is found for NH listeners. Finally, we investigated the effect of electrode separation, as the spread of excitation influences the independence of channels that are physically close together. We compared ITD discrimination thresholds between single-electrode stimulation, three-electrode stimulation with adjacent electrodes, and three-electrode stimulation with electrodes separated by four steps. If the further apart electrodes were more independent, and ITDs are combined across electrodes, improved ITD sensitivity would be expected in this condition.

\section{MATERIALS AND METHODS}

\section{Subjects}

Six subjects bilaterally implanted with Cochlear Nucleus devices participated in the current experiments. They were recruited from the patient population of the University Hospital Leuven (UZ Leuven) and the Medizinische Hochschule Hannover. All subjects were volunteers and signed an informed consent form. This study was approved by the local medical ethical committee. Their travel expenses were reimbursed. Subject details are shown in Table 1.

\section{Apparatus}

The subjects' own sound processors were not used. All stimuli were presented under direct computer control using the APEX 3 program developed at ExpORL, KU Leuven (Francart et al. 2008). Stimulation was done using the Cochlear NICv2 interface connected to two L34 experimental sound processors provided by Cochlear Ltd. with synchronized clocks. Synchronization and stimuli were checked on an oscilloscope before the experiments were started.

\section{Stimuli}

Electrodograms of the stimuli used in the present study are shown in Figures 1 and 2. All stimuli consisted of 1000-pulseper-second (pps) pulse trains modulated with a half-wave rectified 100-Hz sinusoid (Bernstein \& Trahiotis 2002). Electric pulses were presented in monopolar mode $(\mathrm{MP} 1+2)$ and had a phase duration of $25 \mu \mathrm{s}$ with an interphase gap of $8 \mu \mathrm{s}$.

Single-electrode and three-electrode stimuli were used. In three-electrode case, the stimulus conditions differed in relative 
TABLE 1. Subject details

\begin{tabular}{|c|c|c|c|c|c|c|c|}
\hline \multirow[b]{2}{*}{ Subject } & \multirow[b]{2}{*}{ Age (yrs) } & \multirow[b]{2}{*}{ Gender } & \multicolumn{2}{|c|}{ Duration of $\mathrm{Cl}$ Use } & \multirow[b]{2}{*}{ Etiology } & \multicolumn{2}{|c|}{ Cl Type } \\
\hline & & & Left & Right & & Left & Right \\
\hline S1 & 55 & $\mathrm{~F}$ & 0 y $4 \mathrm{~m}$ & 1 y 3 m & Sudden hearing loss & Nucleus $\mathrm{Cl} 422$ & Nucleus $\mathrm{Cl} 422$ \\
\hline S2 & 49 & $\mathrm{~F}$ & 2 y 3 m & 2 y $10 \mathrm{~m}$ & Sudden hearing loss & Nucleus Cl422 & Nucleus Cl422 \\
\hline S3 & 53 & $\mathrm{~F}$ & 5 y $10 \mathrm{~m}$ & 4 y 9 m & Unknown & $\begin{array}{r}\text { Nucleus Cl24RE } \\
\text { (Contour Adv) }\end{array}$ & $\begin{array}{r}\text { Nucleus Cl24RE } \\
\text { (Contour Adv) }\end{array}$ \\
\hline S4 & 56 & $\mathrm{~F}$ & $2 \mathrm{y} 11 \mathrm{~m}$ & 1 y $1 \mathrm{~m}$ & Genetic & Nucleus 24 & Nucleus Cl512 \\
\hline S5 & 65 & M & 13 y $6 \mathrm{~m}$ & 13 y $6 \mathrm{~m}$ & Sudden hearing loss & $\begin{array}{r}\text { Nucleus } 24 \\
\text { (Contour) }\end{array}$ & $\begin{array}{l}\text { Nucleus } 24 \\
\text { (Contour) }\end{array}$ \\
\hline S6 & 22 & $\mathrm{~F}$ & 16 y $2 \mathrm{~m}$ & 12 y $1 \mathrm{~m}$ & $\begin{array}{l}\text { Large vestibular aqueduct } \\
\text { syndrome. }\end{array}$ & $\begin{array}{r}\text { Nucleus } 24 \\
\text { (Contour) }\end{array}$ & $\begin{array}{r}\text { Nucleus } 24 \\
\text { (Contour) }\end{array}$ \\
\hline
\end{tabular}

Duration of $\mathrm{Cl}$ use is given in years $(y)$ and months $(\mathrm{m})$ at the start of the study. Subject S6 was first implanted with a Laura Flex implant and re-implanted with a Nucleus 24 at the age of 9 yrs in the right ear.

delay of the envelope modulation across electrodes. The carrier pulse trains on the three electrodes were as similar as possible, but as stimulating multiple electrodes simultaneously is not possible with the Cochlear Nucleus CI, they were delayed respective to each other by $71 \mu \mathrm{s}$, which is the lowest value technically possible. A the focus of the present study was on the perception of ongoing temporal ITD cues, and the availability of onset ITD cues was minimized by flanking all stimuli by linear onset and offset ramps of $80 \mathrm{~ms}$. Note that the ramps were not additionally modulated (Fig. 1). The total stimulus duration including flanks was $660 \mathrm{~ms}$.

Figure 1 shows the stimuli of the different conditions tested in the present study. Figure 2 shows the same, but zoomed in so that individual pulses become visible. Seven conditions were tested, one single-electrode condition on electrode 13 (e13), and six 3-electrode conditions with electrode 13 in the middle. Of the multiple-electrode conditions, four were on electrode-set 12, 13, and 14 (adjacent electrodes)*. These consisted of one

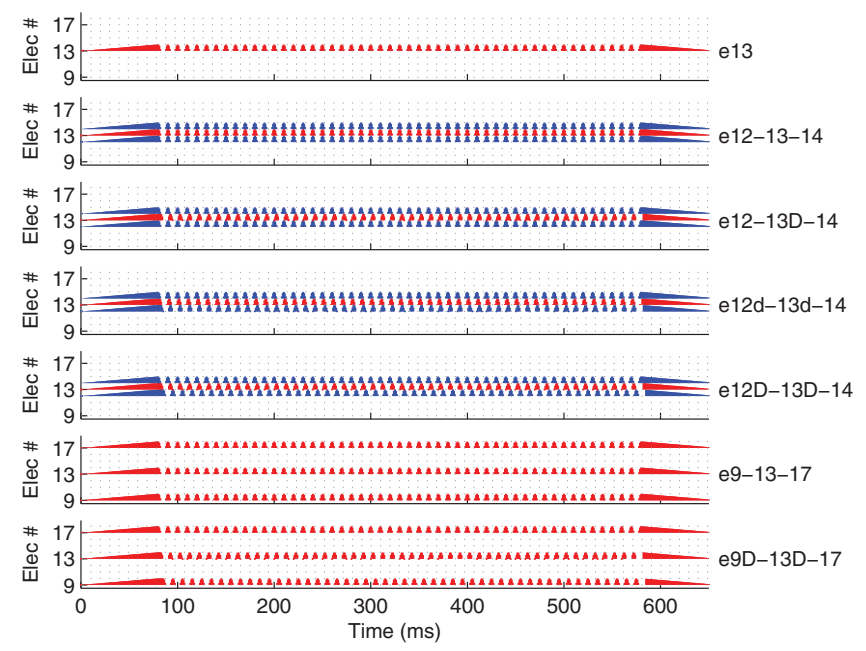

Figure 1. Electrodograms of the stimuli used in the current experiments. The vertical axes indicate the electrode, numbered from apex (1) to base (22). The height of each vertical line represents the magnitude of a biphasic pulse. The colors are used to visually distinguish electrodes with odd and even numbers and have no additional meaning. $D$ and $d$, respectively, signify an interchannel delay of 2.5 and $1.25 \mathrm{~ms}$.

\footnotetext{
* For subject S2, a different selection of electrodes was used. Due to an uncomfortable sensation with stimulation of electrode 14 at C-level, all stimuli were shifted one electrode toward the apex.
}

condition (e12-13-14) in which the envelopes were synchronous across electrodes and three asynchronous conditions. An overview of the stimulus parameters for each condition is given in Table 2. In the first asynchronous condition, the envelope on the middle electrode was delayed by $2.5 \mathrm{~ms}$ relative to electrode 12 (e12-13D-14), in the second asynchronous condition the middle electrode was delayed by $2.5 \mathrm{~ms}$, and the most apical electrode by $5 \mathrm{~ms}$ (e12D-13D-14). The third asynchronous condition was similar to e12D-13D-14, but now with a smaller delay of $1.25 \mathrm{~ms}$ for the middle electrode and $2.5 \mathrm{~ms}$ for the most apical electrode (e12d-13d-14). The synchronous and second asynchronous conditions were additionally tested with a larger electrode separation in conditions e9-13-17 and e9D-13D-17.

When stimuli were presented to the two ears simultaneously, the pulse timing of the stimuli presented to each ear was always identical, and the entire stimulus in one ear was delayed according to the desired ITD, yielding the same ITD in each left-right electrode channel, so no binaural interference is expected.

\section{Procedure}

The procedures consisted of loudness balancing and determination of the just noticeable difference (JND) in an ITD discrimination experiment. ITD sensitivity can depend on the total loudness of a left-right electrode pair (Laback et al. 2011), so

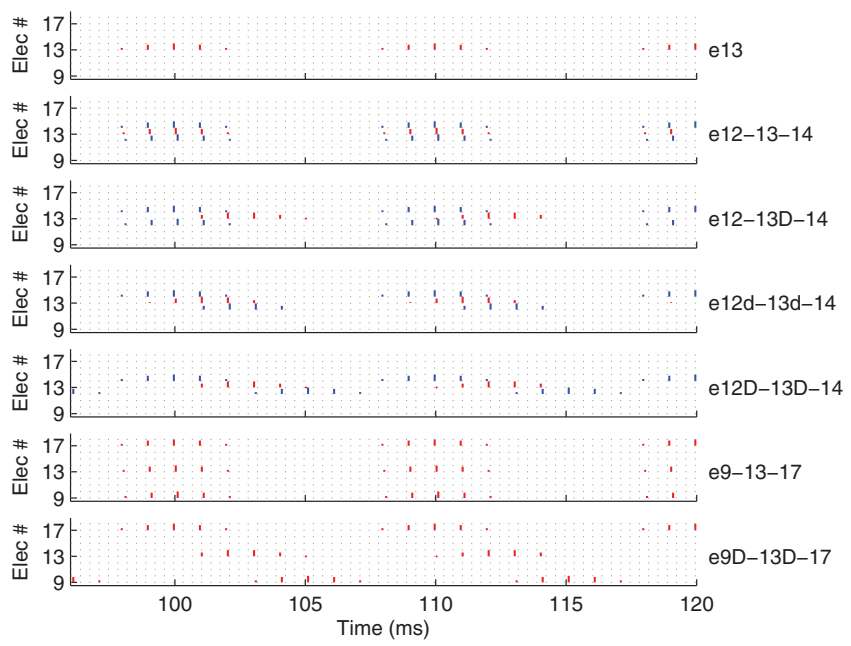

Figure 2. Excerpt of the stimuli used in the current experiments. D and d, respectively, signify an interchannel delay of 2.5 and $1.25 \mathrm{~ms}$. 
TABLE 2. Overview of stimulus parameters

\begin{tabular}{lccccc}
\hline Condition & $\mathrm{e}_{1}$ & $\mathrm{e}_{2}$ & $\mathrm{e}_{3}$ & $\Delta_{1}$ & $\Delta_{3}$ \\
\hline $\mathrm{e} 13$ & 13 & & & $0.00 \mathrm{~ms}$ & $0.00 \mathrm{~ms}$ \\
e12-13-14 & 12 & 13 & 14 & $0.00 \mathrm{~ms}$ & $0.00 \mathrm{~ms}$ \\
e12-13d-14 & 12 & 13 & 14 & $0.00 \mathrm{~ms}$ & $0.00 \mathrm{~ms}$ \\
e12-13D-14 & 12 & 13 & 14 & $0.00 \mathrm{~ms}$ & $0.00 \mathrm{~ms}$ \\
e12D-13D-14 & 12 & 13 & 14 & $5.00 \mathrm{~ms}$ & $0.00 \mathrm{~ms}$ \\
e9-13-17 & 9 & 13 & 17 & $5.00 \mathrm{~ms}$ & $0.00 \mathrm{~ms}$ \\
e9D-13D-17 & 9 & 13 & 17 & $0.00 \mathrm{~ms}$
\end{tabular}

$e_{n^{\prime}}$, the electrode number; $\Delta_{n^{\prime}}$, the envelope delay relative to the electrodes with delay 0.

loudness balancing is needed to avoid this influencing on the one hand the comparison between conditions and on the other hand the relative contribution of each left-right electrode pair in the three-electrode stimuli. In addition, ITD sensitivity is best when interaural level differences do not contradict; therefore, binaural loudness balancing was used to make the interaural level difference as small as possible.

\section{Loudness Balancing}

First threshold (T) and comfortable (C) stimulation levels were determined for every electrode used in the present study, using a 0.5 -s long 1000-pps unmodulated pulse train to determine the dynamic range. Then balancing experiments were performed, first between electrodes monaurally and then across ears.

The modulation depth of the signals for the left and right ear was fixed at the average dynamic range determined for electrodes 12,13 , and 14 in each ear. The $\mathrm{C}$ level for the single-electrode transposed stimulus (e13) was determined in the left ear. The level on the other electrodes for the left ear was balanced with the $\mathrm{C}$ level on electrode 13 . The balancing was done in an adjustment task. Two electrodes were stimulated sequentially, and the listener could adjust the level of the second stimulus until the two intervals sounded equally loud. Listeners were presented with a computer screen with six buttons marked,,,++++++- , -- , and --- corresponding to adjustments of 1, 3, or 6 current units (CUs). Subjects confirmed the balancing by pressing a stop button in the center of the screen. Two runs were performed for the same electrode pair. One run started well below the balanced level, and the other started above the balanced level; the average was used as the balanced level. Listeners were encouraged to adjust the level to above and below the balanced level before they confirmed the balanced level. In the next step, the three-electrode stimuli were balanced in loudness with the stimulus on electrode 13 in a similar fashion. In this step, the relative levels of the electrodes were fixed, that is, the difference between the electrodes in CUs was fixed, and the levels on the three electrodes were adjusted simultaneously, in equal current steps for each electrode.

All previous steps were done monaurally in the left ear. Next, the levels were balanced between the ears. The balancing between the left and right ear was done in a centring task. The stimuli were presented simultaneously to both ears (ITD $=0 \mu \mathrm{s}$ ), and the subjects were asked to adjust the level in the right ear so the sound was perceived in the center of the head. Subjects were encouraged to move the sound image to both sides before confirming it was centered. First, all single-electrode stimuli in the right ear were balanced with the level previously determined on the same electrode in the left ear. Then for the three-electrode stimulus, the relative levels determined on the individual electrodes were kept fixed, and the overall stimulus level was adjusted to balance it with the left ear by adding or subtracting a fixed number of CUs to the pulse train on each electrode. This step was also performed two times, and started one time with the level in the right ear below the level in the left ear, and one time the other way around. The average was used as the balanced level.

At the start of every session, the centralization was checked before measuring the JND in ITD. This step was included because there have been reports in the literature that binaural balance can be unstable in CI subjects. This was done using a left/center/right task, playing two equally large ITDs at both sides and one with ITD zero. If the sound image was not heard in the center and did not move equally far to either side, the rightear level was adjusted to correct for the asymmetry and the procedure was repeated. As a final check, several ITDs were played in increasing or decreasing order, from one side of the head to the other, choosing stimuli symmetrically around ITD $0 \mu \mathrm{s}$. The subject was asked to indicate whether they could hear the sound image moving from one side to the other and whether the extent of lateralization was similar at both sides. If not, the balancing procedure was repeated.

\section{ITD Discrimination}

As the subjects were unfamiliar with the ITD discrimination task in the present study, before the actual data was collected, they were familiarized with the task. During familiarization, the single-electrode stimulus with various ITDs was presented manually by the experimenter until the subject could reliably lateralize based on ITD. Then, the JND in ITD was determined with an adaptive procedure until a stable response (convergent staircase) was obtained. The latter adaptive procedure was identical to the one used in the actual ITD discrimination experiment, but the results were not retained for analysis.

In the ITD discrimination experiment, JNDs in ITD were determined using an adaptive one-up-two-down, two-interval, two-alternative-forced-choice procedure. The two intervals contained an equally large ITD of opposite sign. Listeners were asked whether the second interval sounded left or right of the first. The procedure was started at an ITD of $1800 \mu \mathrm{s}$, and the ITD was adjusted by factor 1.58 for the first two reversals, and thereafter by factor 1.26. The procedure was stopped after 10 reversals. The JNDs were calculated as the geometric mean of the last eight reversals. Each condition was tested several times over multiple test sessions. While we aimed for six runs of the adaptive procedure per condition, due to subject-time constraints this was not possible in all cases. Data were only included for conditions where at least three runs were available. The final JND was calculated as the geometric mean of the 
thresholds obtained in each run (three up to six). Except for the single-electrode stimulus, which was used in the familiarization phase and in the first actual test session, stimulus conditions were randomly distributed across test sessions, differently for each subject. In total, nine sessions of approximately 2 hours were needed for subject S4, six sessions for subject S1, S2, and S6, and five sessions for subject S3 and S5 to collect the data. For subjects S3 and S6, not all conditions could be tested.

\section{Statistical Analysis}

Before statistical analysis, the JND in ITD data were logarithmically transformed. For conditions e12d-13d-14 and e1213D-14, data are missing for one and two subjects, respectively, due to testing time constraints. Therefore, no statistical analysis was performed on the entire data set, but instead different research questions were tested individually, based on the available data. For each research question, a repeated measures analysis of variance (RM-ANOVA) of JND in ITD was conducted using SPSS.

\section{RESULTS}

Figures 3 and 4 show the JND in ITD for every condition per subject. The horizontal axis shows subject codes, the logarithmic vertical axis the JND in ITD, and different colors indicate the conditions shown in the legend. In Figure 3, the various synchronous conditions are compared (e13, e12-13-14, e9-13-17). In Figure 4, the synchronous and asynchronous conditions are compared for (a) neighboring electrodes, and (b) electrodes four steps apart.

\section{Effect of Single Versus 3-Electrode Stimulation}

To investigate the effect of single- versus multiple-electrode stimulation and of electrode spacing (Fig. 3), an RM-ANOVA of condition was performed for the single-electrode (e13) and synchronous multiple-electrode conditions (e12-13-14, e9-13-17), for all six subjects. There was no significant effect $(F=0.645$, $p=0.545)$. Overall, two subject groups could be distinguished: the good performers (S1, S2, S3, and S4) with single-electrode JNDs in ITD (condition e13) lower than $700 \mu \mathrm{s}$, and a group of poor performers (S5, S6) with JNDs outside the physically relevant range of $700 \mu \mathrm{s} . \dagger$ As for the poor performers on the one hand, potential effects may be obscured by a ceiling effect and on the other hand their single-channel thresholds were outside the physically realistic range, we conducted the same analysis excluding the poor performers and similarly found no effect of single versus multiple-electrode stimulation or electrode spacing $(F=2,209, p=0.191)$.

In summary, no significant effect of single versus multiple electrodes was found nor an effect of electrode spacing.

\section{Effect of Synchronous Versus Asynchronous Channel Envelopes}

To investigate the effect of synchronous versus asynchronous channel envelopes in the adjacent-electrode conditions, we compared conditions e12-13-14, e12-13D-14, e12d-13d-14, and e12D-13D-14. From Figure 4A, it can be seen that for all subjects there was a decrease in sensitivity in the asynchronous conditions compared with the

\footnotetext{
$\uparrow$ Due to the size of the human head, naturally ITDs occur in the range of
} -700 to $700 \mu \mathrm{s}$.

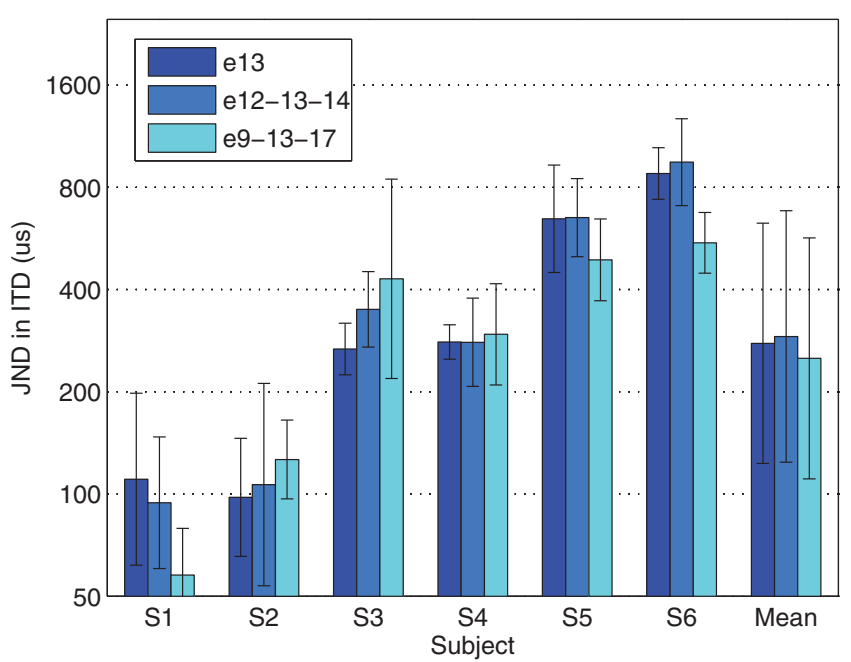

Figure 3. Mean JND in ITD for the synchronous conditions with electrodes 13, 12-13-14, or 9-13-17. The error bars represent geometric standard deviations. ITD, interaural time difference; JND, just noticeable difference.

synchronous condition. An RM-ANOVA of condition (e1213-14/e12-13D-14/e12d-13d-14/e12D-13D-14) for the four subjects for whom data were available in these four conditions (S1, S2, S4, S5) yielded a significant effect of asynchrony $(F=7.408, p=0.008)$. No significant pairwise effects were found. When considering only the good performers for whom data were available in these four conditions (S1, S2, S4), a significant effect of asynchrony was found $(F=20.831, p<0.001)$ and in paired comparisons also significant differences were found between conditions e12-13-14 and e12D-13D-14 ( $p=0.036)$ and between conditions e12d-13d-14 and e12D-13D-14 ( $p=0.020)$.

In summary, the JND ITD increased on average from 291 to $1109 \mu$ s when introducing $2.5 \mathrm{~ms}$ delays on two electrodes (condition e12D-13D-14), but there was no clinically meaningful difference (average from $291 \mu$ s to 376 or $245 \mu$ s) for smaller delays.

\section{Effect of Electrode Separation and Synchrony}

To investigate the effect of synchronous versus asynchronous channel envelopes in the spaced electrode conditions, we compared conditions e9-13-17 and e9D-13D-17 in an RM-ANOVA (Fig. 4B). For all subjects together, there was no effect of synchrony $(p=0.17)$. For the good performers, it was on the edge of significance $(F=17.269, p=0.053)$.

Finally, the combined effect of electrode separation and (a) synchrony was investigated by conducting an RM-ANOVA with factors electrode separation, asynchrony, and their interaction for conditions e12-13-14, e9-13-17, e12D-13D-14, and e9D-13D-17. Overall, there was a significant sensitivity drop with asynchrony $(F=22.85, p=0.005)$, no effect of separation $(F=3.48, p=0.121)$, and no significant interaction $(F=2.196$, $p=0.198)$. A paired $t$ test between condition e12D-13D-14 and e9D-13D-17 indicated no significant effect $(t=1.743$, $p=0.129$ ). Similarly, including only the good performers (S1$4)$, there was a performance drop with asynchrony $(F=15.54$, $p=0.029)$, no effect of separation $(F=1.76, p=0.276)$, and no interaction $(F=4.899, p=0.114)$.

In summary, again there was a large ITD sensitivity drop when introducing $2.5 \mathrm{~ms}$ delays on two electrodes, but now in 

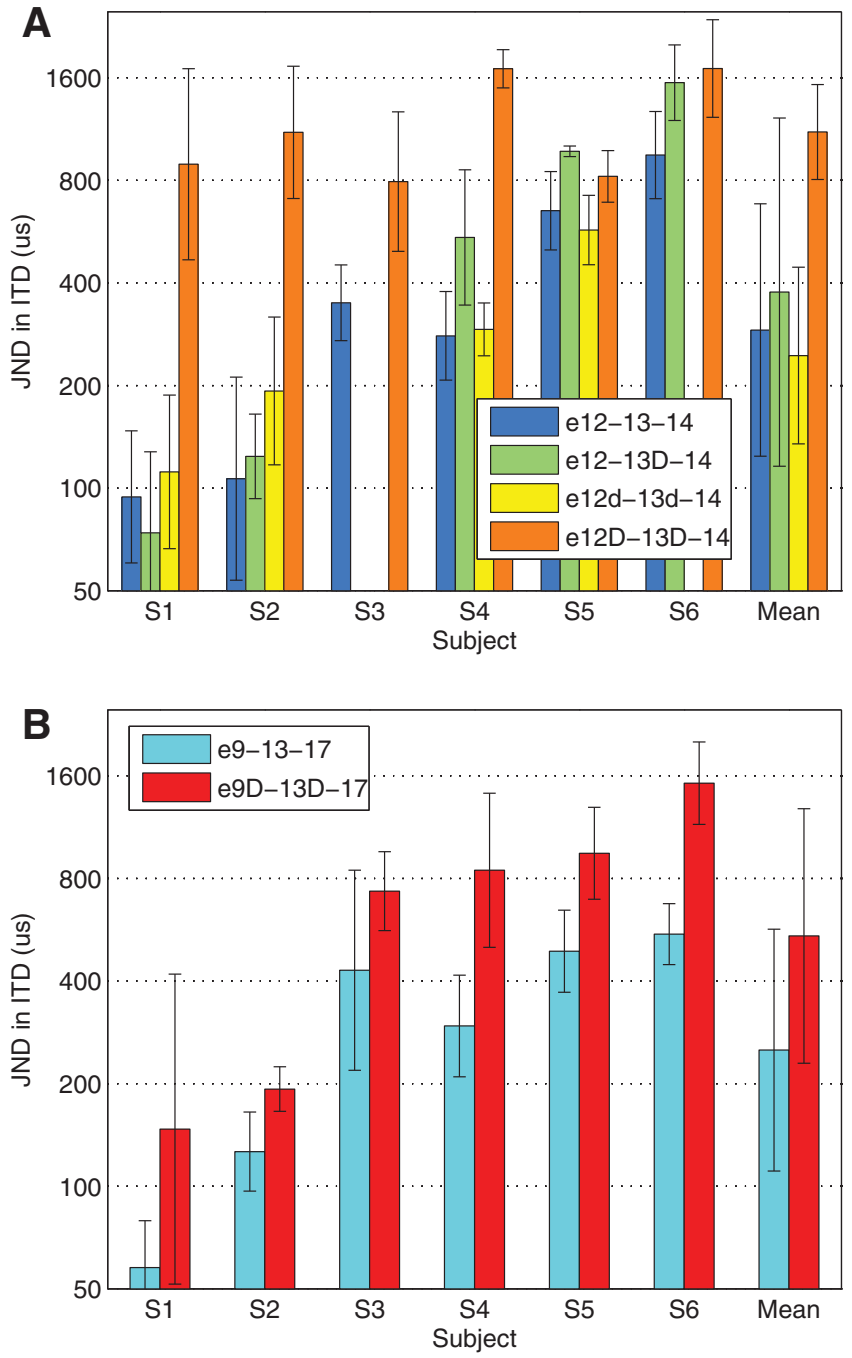

Figure 4. A and B, Mean JND in ITD in various delay conditions, with $\mathrm{d}=1.25 \mathrm{~ms}$ and $\mathrm{D}=2.5 \mathrm{~ms}$. The error bars represent geometric standard deviations. ITD, interaural time difference; JND, just noticeable difference.

the further spaced electrode condition (e9D-13D-17). There was no significant effect of electrode separation.

\section{DISCUSSION}

While many bilateral CI users are sensitive to ITDs with wellcontrolled stimuli in the laboratory, they do not use ITDs for many sounds presented through their clinical processors. Due to the effects of room acoustics and the filter banks and other signal processing implemented in current clinical processors, there can be delays (asynchrony) between the envelope modulations in different electrode channels. We investigated the effect of electrode spacing and electrode delay on ITD sensitivity with bilateral CI users listening to single- and three-channel pulse trains. We found that ITD sensitivity significantly decreased with increasing acrosschannel delay but found no significant effect of electrode spacing.

\section{Across-Subject Differences}

The results show large differences in overall sensitivity level of the subjects, which is consistent with the literature (e.g., Figure 5, including data from van Hoesel et al. 2009; Best et al. 2011; Laback et al. 2011; Kerber \& Seeber 2013; Noel \& Eddington 2013). Also note that in some studies listeners are preselected based on ITD sensitivity. While there is no clear explanation yet why such large intersubject differences are found, it has been suggested that it could be due to variation in asymmetry in neural survival between the ears. Another factor that has been shown to play a role is the age at the time of implantation. In a comparative study, Litovsky et al. (2010) found that ITD sensitivity for prelingually deafened bilateral CI users was much lower or even absent, whereas the bilateral CI users with adult onset of deafness all showed sensitivity to ITD to some extent. Our subject S6 was prelingually deafened and received her second implant 4 years after the first one. She had bilateral deprivation until she received her first CI and a lack of binaural input for several years until she received her second CI, which can negatively impact ITD sensitivity (Poon et al. 2009). The other subjects had adult onset of deafness and access to binaural acoustic hearing before implantation. Note that we obtained some JNDs much larger than the maximal physically available ITD (around $700 \mu$ s), and such thresholds could have been considered unmeasurable in other studies.

\section{Combination of ITD Cues Across Channels}

Comparison of the single-electrode conditions with the synchronous three-electrode conditions did not indicate a significant effect of introducing the same ITD on multiple electrodes. On the individual level, for subjects S1 and S6, compared with condition e12-13-14, there was a tendency of better sensitivity in condition e9-13-17. If this was not due to chance, it is unclear whether it was due to better sensitivity for one of the individual electrodes (9 or 17), or whether it was an effect of across-channel ITD summation (Dye 1990; Buell \& Trahiotis 1993; Saberi 1995), which would indicate that increasing the separation between the stimulated electrodes led to more information being independently transmitted. Ihlefeld et al. (2014) investigated the effect of dual- versus single-electrode stimulation on ITD sensitivity but for left-right electrode pairs spaced further apart. They compared ITD sensitivity on apical, basal, and apical + basal electrode pairs and found that for apical + basal sensitivity was not worse compared with the best of the apical or basal pairs.

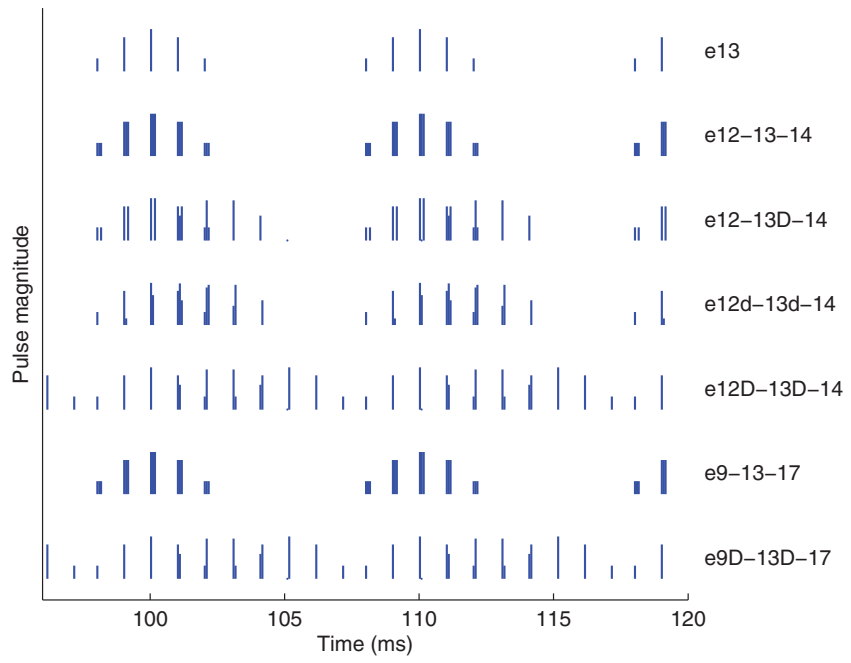

Figure 5. Excerpt of the aggregate pulse pattern for each condition. D and d, respectively, signify an interchannel delay of 2.5 and $1.25 \mathrm{~ms}$. 
That is, similar to our data, they found no direct evidence of binaural interference or ITD summation.

Comparing between conditions e12D-13D-14 and e9D$13 \mathrm{D}-17$, while the overall difference was not significant, thresholds deceased for all listeners except S5, with thresholds for e9D-13D-17 back in the range of the nondelayed conditions for $\mathrm{S} 1, \mathrm{~S} 2$, and S4. This suggests that for asynchronous envelopes, it can be beneficial if electrodes are spaced further apart. In this case, the envelopes could be more independently processed and combined by the binaural system rather than the binaural system using the aggregate across-channel envelope.

Differences in sensitivity with changing electrode distance have been demonstrated in the literature for other tasks than ITD discrimination: modulation detection, pitch ranking, and comodulation masking release. Chatterjee and Oba (2004) showed significant across-electrode interactions in modulation detection experiments for electrodes that were separated by up to $12 \mathrm{~mm}$ (16 electrodes). In pitch ranking experiments, McKay and McDermott (1996) found that the pitch perceived on dual electrode stimuli was similar to the pitch evoked by the aggregate stimulation pattern when stimulated electrodes were separated less than three to four-electrode distances. Pierzycki and Seeber (2014) found that increasing electrode separation increased the effect of comodulation release of masking with electric stimulation.

\section{Effect of Synchronous Versus Asynchronous Channel Envelopes}

Comparing the adjacent-electrode conditions with various delays, there was on average no clinically meaningful difference in ITD sensitivity, except for e12D-13D-14, where sensitivity was much lower, with ITD thresholds larger than the physical limit of $700 \mu \mathrm{s}$. If the three electrodes stimulated overlapping populations of neurons, the reduced sensitivity in the asynchronous conditions could be explained by the acrosselectrode summed stimulation patterns shown in Figure 5. In conditions e12-13D-14 and e12d-13d-14, the aggregate waveform has a $100-\mathrm{Hz}$ periodicity and some dead time before each cycle. In condition e12D-13D-14, the aggregate waveform has no remaining dead time and additional $300-\mathrm{Hz}$ periodicity, both factors that can reduce ITD sensitivity (Laback et al. 2011; Noel \& Eddington 2013). This means that in the adjacent-electrode conditions, the three electrodes did probably not function as separately processed information channels, presumably due to spread of excitation. In the condition with electrodes 9-13-17, that is, larger electrode spacing than $12-13-14$, the same tendency of worse sensitivity is present, but thresholds in the e9D-13D-17 condition tend to be lower than in the e12D-13D-14 condition, suggesting that here the auditory system could at least to some extent use information from separate information channels.

\section{Consequences for Sound Processors}

Due to the effect of room acoustics and the filter banks, maximal selection and other processing implemented in commercial sound processors, for periodic input sounds there are often large asynchronies across-channel envelopes, up to half the period of the acoustic signal. Delays in this order of magnitude were tested in the present study. While apart from these asynchronies there are other problems to be solved to enable good ITD sensitivity with commercial sound processors, it follows from our results that to enhance ITD sensitivity with bilateral CIs, envelopes presented to electrodes close together should ideally be synchronously modulated, allowing for some dead time in the aggregate stimulation pattern. However, ITD sensitivity was resilient to small delays, as long as the aggregate stimulation pattern contained clear modulations. In practice, this could be achieved in a sound processor by using either a single modulation waveform to modulate all channel envelopes, such as in the Modulation Enhancement Strategy (Francart et al. 2014), or using overlapping filters in the filter bank from which the envelopes are extracted, which may be optimized as a good compromise between providing sufficiently comodulated signals and providing separate information channels.

\section{CONCLUSIONS}

In summary, for synchronous envelopes we found no effect on ITD sensitivity for single- versus three-electrode stimulation, nor of electrode spacing in two 3-electrode conditions. This means that we found no ITD summation effect, as exhibited by $\mathrm{NH}$ listeners when presented with the same ITD in different tonotopic channels.

For asynchronous envelopes, we found reduced ITD sensitivity with increasing asynchrony, especially when stimulating adjacent electrodes. This sensitivity reduction is likely caused by reduction in off-time between the active portions of every modulation cycle in the aggregate stimulation pattern. Again no statistically significant ITD summation effect was found.

For the development of stimulation strategies that enable the use of ITDs, this means that to some extent envelopes presented to electrodes close together should be synchronously modulated, of course without compromising other aspects such as speech intelligibility.

\section{ACKNOWLEDGMENTS}

The authors thank the staff of the audiology team of the MH-Hannover and the University Hospital of Leuven for their support. We are grateful to our test subjects, who spent many hours patiently listening to the same stimuli all over again. This study was performed as part of the Agency for Innovation by Science and Technology project number 110722 in cooperation with Cochlear Ltd. Anneke Lenssen was sponsored by the European Union-Initial Training Network project AUDIS, grant agreement number PITN-GA-2008-214699.

T. F. and A. L. declare equal contributions to this study and should be considered joint first authors.

The authors declare no conflict of interest.

Address for correspondence: Tom Francart, ExpORL, Department of Neurosciences, KU Leuven, O \& N 2, Herestraat 49 bus 721, B-3000 Leuven, Belgium. E-mail: tom.francart@med.kuleuven.be

Received June 17, 2014; accepted January 23, 2015.

\section{REFERENCES}

Bernstein, L. R., \& Trahiotis, C. (2002). Enhancing sensitivity to interaural delays at high frequencies by using "transposed stimuli." J Acoust Soc Am, 112(3 Pt 1), 1026-1036.

Best, V., Laback, B., Majdak, P. (2011). Binaural interference in bilateral cochlear-implant listeners. J Acoust Soc Am, 130, 2939-2950. 
Buell, T. N., \& Trahiotis, C. (1993). Interaural temporal discrimination using two sinusoidally amplitude-modulated, high-frequency tones: Conditions of summation and interference. J Acoust Soc Am, 93, 480-487.

Buell, T. N., Trahiotis, C., Bernstein, L. R. (1991). Lateralization of low-frequency tones: Relative potency of gating and ongoing interaural delays. $J$ Acoust Soc Am, 90, 3077-3085.

Chatterjee, M., \& Oba, S. I. (2004). Across- and within-channel envelope interactions in cochlear implant listeners. J Assoc Res Otolaryngol, 5, $360-375$.

Ching, T. Y., van Wanrooy, E., Dillon, H. (2007). Binaural-bimodal fitting or bilateral implantation for managing severe to profound deafness: A review. Trends Amplif, 11, 161-192.

Dye, R. J. (1990). The combination of interaural information across frequencies: Lateralization on the basis of interaural delay. $J$ Acoust Soc Am, 88, 2159-2170.

Francart, T., Lenssen, A., Wouters, J. (2014). Modulation enhancement in the electrical signal improves perception of interaural time differences with bimodal stimulation. J Assoc Res Otolaryngol, 15, 633-647.

Francart, T., van Wieringen, A., Wouters, J. (2008). APEX 3: A multi-purpose test platform for auditory psychophysical experiments. $J$ Neurosci Methods, 172, 283-293.

Grantham, D. W., Ashmead, D. H., Ricketts, T. A., et al. (2008). Interaural time and level difference thresholds for acoustically presented signals in post-lingually deafened adults fitted with bilateral cochlear implants using CIS+ processing. Ear Hear, 29, 33-44.

Heller, L. M., \& Trahiotis, C. (1995). Interference in detection of interaural delay in a sinusoidally amplitude-modulated tone produced by a second, spectrally remote sinusoidally amplitude-modulated tone. J Acoust Soc Am, 97, 1808-1816.

Ihlefeld, A., Kan, A., Litovsky, R. Y. (2014). Across-frequency combination of interaural time difference in bilateral cochlear implant listeners. Front Syst Neurosci, 8, 22.

Kerber, S., \& Seeber, B. U. (2013). Localization in reverberation with cochlear implants: Predicting performance from basic psychophysical measures. J Assoc Res Otolaryngol, 14, 379-392.

Laback, B., Pok, S. M., Baumgartner, W. D., et al. (2004). Sensitivity to interaural level and envelope time differences of two bilateral cochlear implant listeners using clinical sound processors. Ear Hear, 25, 488-500.

Laback, B., Zimmerman, I., Majdak, P., et al. (2011). Effects of envelope shape on interaural envelope delay sensitivity in acoustic and electric hearing. J Acoust Soc Am, 130, 1515-1529.

Litovsky, R. Y., Jones, G. L., Agrawal, S., et al. (2010). Effect of age at onset of deafness on binaural sensitivity in electric hearing in humans. J Acoust Soc Am, 127, 400-414.
Macherey, O., \& Carlyon, R. P. (2010). Temporal pitch percepts elicited by dual-channel stimulation of a cochlear implant. J Acoust Soc Am, 127, 339-349.

Majdak, P., Laback, B., Baumgartner, W. D. (2006). Effects of interaural time differences in fine structure and envelope on lateral discrimination in electric hearing. J Acoust Soc Am, 120, 2190-2201.

McKay, C. M., \& McDermott, H. J. (1996). The perception of temporal patterns for electrical stimulation presented at one or two intracochlear sites. $J$ Acoust Soc Am, 100(2 Pt 1), 1081-1092.

McKay, C. M., \& McDermott, H. J. (1999). The perceptual effects of current pulse duration in electrical stimulation of the auditory nerve. $J$ Acoust Soc Am, 106, 998-1009.

Noel, V. A., \& Eddington, D. K. (2013). Sensitivity of bilateral cochlear implant users to fine-structure and envelope interaural time differences. $J$ Acoust Soc Am, 133, 2314-2328.

Pierzycki, R. H., \& Seeber, B. U. (2014). Comodulation masking release in electric hearing. J Assoc Res Otolaryngol, 15, 279-291.

Poon, B. B., Eddington, D. K., Noel, V., et al. (2009). Sensitivity to interaural time difference with bilateral cochlear implants: Development over time and effect of interaural electrode spacing. J Acoust Soc Am, 126, 806-815.

Saberi, K. (1995). Lateralization of comodulated complex waveforms. $J$ Acoust Soc Am, 98, 3146-3156.

Schafer, E. C., Amlani, A. M., Paiva, D., et al. (2011). A meta-analysis to compare speech recognition in noise with bilateral cochlear implants and bimodal stimulation. Int $J$ Audiol, 50, 871-880.

Seeber, B. U., \& Fastl, H. (2008). Localization cues with bilateral cochlear implants. J Acoust Soc Am, 123, 1030-1042.

Senn, P., Kompis, M., Vischer, M., et al. (2005). Minimum audible angle, just noticeable interaural differences and speech intelligibility with bilateral cochlear implants using clinical speech processors. Audiol Neurootol, 10, 342-352.

Stellmack, M. A. (1992). Detection of an interaural delay of a reproducible target noise band in the presence of reproducible diotic noise bands. $J$ Acoust Soc Am, 92, 2363.

Tong, Y. C., \& Clark, G. M. (1986). Loudness summation, masking, and temporal interaction for sensations produced by electric stimulation of two sites in the human cochlea. J Acoust Soc Am, 79, 1958-1966.

van Hoesel, R. J. (2007). Sensitivity to binaural timing in bilateral cochlear implant users. J Acoust Soc Am, 121, 2192-2206.

van Hoesel, R. J., Jones, G. L., Litovsky, R. Y. (2009). Interaural time-delay sensitivity in bilateral cochlear implant users: Effects of pulse rate, modulation rate, and place of stimulation. J Assoc Res Otolaryngol, 10, 557-567.

van Hoesel, R. J., \& Tyler, R. S. (2003). Speech perception, localization, and lateralization with bilateral cochlear implants. J Acoust Soc Am, 113, 1617-1630. 